\title{
Resonant Diffraction Radiation and Smith-Purcell Effect
}

\author{
A.P.Potylitsin \\ Nuclear Physics Institute, \\ Tomsk Polytechnic University, \\ pr. Lenina 2A, P.O.Box 25, \\ 634050 Tomsk, Russia \\ e-mail: pap@phtd.tpu.edu.ru
}

\begin{abstract}
An approach has been developed where the Smith-Purcell radiation (SPR), i.e. emission of electrons moving close to a periodic structure, is treated as the resonant diffraction radiation. Simple formulas have been designed for the SPR intensity for a grating having perfectly conducting strips spaced by a vacuum gap. The results have been compared with those obtained via other techniques. It has been shown that the intensity of radiation for the said gratings for a relativistic case sufficiently exceeds the SPR intensity for the grating made up by a periodically deformed continuous surface.
\end{abstract}


Radiation of electrons passing in the vicinity of a periodic structure made of some conducting material, the so-called Smith-Purcell effect (SPE), has been studied by both experimental and theoretical scholars for over 40 years now since its discovery. Nonetheless, only the recent years saw the first experiments using relativistic beams [1-3]. Design and realization of such experiments followed by comparison with numerical calculations (see e.g. Refs.4 and 5) is retarded due to the absence of a simple physical model capable of accounting for the emission characteristics in terms of periodic structure parameters. Within the current approach [6,7], based on the scalar diffraction theory, the complexity of numerical calculations, resulting from a merely optical problem of the grating reflectivity on the whole, "overshadows" the physical mechanism of SPE. We believe that the technique proposed by M.Moran [8] is deemed more efficient. The author considers SPE for a stack of semi-infinite foils located normal to the beam trajectory using the radiation characteristics on each of the foil and taking into account interference from all the stack.

The present paper offers a more feasible model treating SPE as the resonant diffraction radiation (RDR) of a relativistic particle on a set of strips of a finite width. Fig.1 shows two different geometries of the strip arrangement: parallel (case $a$ ) and perpendicular (case $b$ ).

Characteristics of the diffraction radiation (DR) of the motion of a charged particle close to a perfectly conducting semi-plane were derived in Ref.9 by solving the Maxwell equations via the Wiener-Hopf method. Following the latter formalism [9], one may write the spectralangular density of DR (neglecting the terms of the $\gamma^{-2}$ order, $\gamma$ being the Lorentz factor) as:

$$
\frac{d^{2} W_{\|}}{d \Omega d \omega}=\frac{\alpha}{2 \pi^{2}} \frac{\exp \left(-\frac{\omega}{\omega_{c}} \sqrt{1+\gamma^{2} \xi^{2} \sin ^{2} \theta}\right)}{1-\cos \theta}
$$

For the case a) and

$$
\frac{d^{2} W_{\perp}}{d \Omega d \omega}=\frac{\alpha}{4 \pi^{2}} \frac{\exp \left(-\frac{\omega}{\omega_{c}} \sqrt{1+\gamma^{2} \xi^{2} \sin ^{2} \theta}\right)}{\sin ^{2} \theta} \frac{1-\sin \theta+2 \gamma^{2} \xi^{2} \sin ^{2} \theta}{1+\gamma^{2} \xi^{2} \sin ^{2} \theta}
$$

for the case b).

The system of units used throughout the paper is $\hbar=m_{e}=c=1$. 
In the expressions (1) and (2) $\alpha=1 / 137$ is the fine structure constant, $\omega_{c}=\frac{\gamma}{2 h}$ is the DR critical energy, $h$ is the impact parameter, $\lambda$ is wavelength of DR, $\omega=\frac{2 \pi}{\lambda}, \theta$ and $\xi$ are the polar $\left(\theta>>\gamma^{-1}\right)$ and azimuthal photon outgoing angles $(\xi<<1$ and is measured from the plane containing the electron momentum and normal to the strip edge).

The DR intensity is determined by the characteristic factor (for $\xi=0$ )

$$
\exp \left(-\frac{\omega}{\omega_{c}}\right)=\exp \left(-\frac{4 \pi h}{\gamma \lambda}\right)
$$

which can be connected with the intensity of virtual photons scattered by the only edge of the semi-plane (the field strength of the virtual photons is determined by the factor $\left.\exp \left(-\frac{2 \pi h}{\gamma \lambda}\right)\right)$.

It can be shown that, with electrons moving parallel to the strip surface, $\mathrm{DR}$ is formed during scattering of the virtual field on the edges of the strip, with

$$
\vec{E}_{\text {exit }}=-\vec{E}_{\text {entrance }} e^{i \varphi}
$$

The interference of the fields (3) is taken into account in a manner similar to the theory of transition radiation (TR) (see e.g. Ref.10):

$$
\frac{d^{2} W_{\|}^{s t}}{d \omega d \Omega}=\frac{d^{2} W_{\|}}{d \omega d \Omega}\left|1-e^{i \varphi_{\|}}\right|^{2}=\frac{d^{2} W_{\|}}{d \omega d \Omega} F_{\|}
$$

The interference factor

$$
F_{\|}=2\left(1-\cos \varphi_{\|}\right)=4 \sin ^{2} \frac{\varphi_{\|}}{2}
$$

is determined by the phase $\varphi_{\|}$that coincides with the corresponding phase for TR of the vacuum slit and large outgoing angles $\left(\theta>>\gamma^{-1}\right)$ :

$$
\varphi_{\|}=\frac{2 \pi a}{\lambda}\left(\frac{1}{\beta}-\cos \theta\right) \approx \frac{2 \pi a}{\lambda}(1-\cos \theta)
$$

In the case $b$ we encounter a more complicated interference with fields of different strength (see Fig.1b):

$$
\frac{d^{2} W_{\perp}^{s t}}{d \omega d \Omega}=\left|\vec{E}_{\perp}\left(h-\frac{a}{2}\right) e^{-i \varphi_{\perp}}-\overrightarrow{E_{\perp}}\left(h+\frac{a}{2}\right) e^{+i \varphi_{\perp}}\right|^{2}=
$$




$$
\begin{gathered}
\left|\overrightarrow{E_{\perp}}(h)\right|^{2}\left|\exp \left(\chi-i \varphi_{\perp}\right)-\exp \left(-\chi+i \varphi_{\perp}\right)\right|^{2}=\frac{d^{2} W_{\perp}}{d \omega d \Omega} F_{\perp} \\
F_{\perp}=4\left(\sin h^{2} \chi+\sin ^{2} \varphi_{\perp}\right)
\end{gathered}
$$

The notation used is as follows:

$$
\begin{gathered}
\chi=\frac{\pi a}{\gamma \lambda} \sqrt{1+\gamma^{2} \xi^{2} \sin ^{2} \theta} \\
\varphi_{\perp}=\frac{\pi a}{\lambda} \sin \theta
\end{gathered}
$$

It should be underlined that the interference factors (5) and (8) are independent of the impact parameter $h$ as expected.

The interference of DR from a grating consisting of $N$ structural elements (RDR) is described in an entirely the same fashion as is the resonant transition radiation $[11]$ :

$$
\begin{aligned}
\frac{d^{2} W_{N}^{\|, \perp}}{d \Omega d \omega} & =\frac{d^{2} W^{\|, \perp}}{d \Omega d \omega} F_{\|, \perp} F_{N}, \\
F_{N} & =\frac{\sin ^{2}\left(N \frac{\varphi}{2}\right)}{\sin ^{2}\left(\frac{\varphi}{2}\right)} .
\end{aligned}
$$

The phase $\varphi$ in Eq. (12) is controlled solely by the grating period $d$ but not its structure:

$$
\varphi=\frac{2 \pi d\left(\cos \theta-\frac{1}{\beta}\right)}{\lambda}
$$

For a considerably large number of elements, $N>>1$, we may use a simpler formula [10]:

$$
F_{N}=2 \pi N \delta(\varphi-2 k \pi)
$$

$k$ is the integer.

The argument of the $\delta$ - function governs the so-called dispersion relation:

$$
\lambda_{k}=\frac{d\left(\cos \theta-\frac{1}{\beta}\right)}{k}
$$


The above expression (15) is derived from general considerations irrespective of any particular radiation mechanism.

The expressions similar to Eq.(15) determine the position of lines in the spectrum radiated not only for SPE but also for RTR and parametric X-ray radiation $[7,11,12]$.

Proceeding from Eqs.(4), (11) and (14), upon integration with respect to frequency we may obtain the angular distribution of the SP radiation calculated per unit cell of the grating.

Consider first the case 1a). As follows from Eq.(5), the maximum yield is attained for the $\varphi_{\|}=\pi$, where from follows the relation between the strip width and the period:

$$
\frac{a}{d}=\frac{1}{2}
$$

Then, for the fundamental diffraction order $(k=-1)$ we have:

$$
\frac{d W_{s p}^{\|}}{d \Omega}=\frac{2 \alpha}{\pi^{2}} \omega_{-1} \frac{\exp \left(-\frac{\omega_{-1}}{\omega_{c}} \sqrt{1+\gamma^{2} \xi^{2} \sin ^{2} \theta}\right)}{1-\cos \theta}
$$

where

$$
\omega_{-1}=\frac{2 \pi}{\lambda_{-1}}=\frac{2 \pi}{d\left(\frac{1}{\beta}-\cos \theta\right)} .
$$

In Ref.6 a similar problem was solved via a different technique, the resulting Eq.(17), however, was rather close to the one in Ref.6, where instead of a two the numerator has the factor $(1+\cos \theta)$.

Fig.2a shows the calculations for the following conditions: $\gamma=100 ; d=$ $6 \mathrm{~mm} ; h=15 \mathrm{~mm} ; \xi=0$.

For the 1b) geometry the SPR angular distribution under the same conditions acquires a somewhat more complicated form:

$$
\frac{d W_{s p}^{\perp}(\xi=0)}{d \Omega}=\frac{\alpha}{\pi^{2}} \exp \left(-\frac{\omega_{-1}}{\omega_{c}}\right) \omega_{-1} \frac{1-\sin \theta}{\sin ^{2} \theta}\left(\sinh ^{2} \chi+\sin ^{2} \varphi_{\perp}\right)
$$

The last term in Eq.(19) has a periodic character with the period being $\Delta \varphi_{\perp}=\pi$. Therefore the distribution Eq.(19) would have dips for the outgoing angles, when $\sin ^{2} \varphi_{\perp}$ becomes zero: 


$$
\varphi_{\perp}=\frac{\pi a}{\lambda_{-1}} \sin \theta=\frac{\pi a \sin \theta}{d(1-\cos \theta)}=m \pi
$$

Hence for $\frac{a}{d}=\frac{1}{2}$ we have $\tan \frac{\theta_{m}}{2}=\frac{1}{2 m}$, and therefore, $\theta_{1}=53.13^{\circ}$; $\theta_{2}=28.07^{\circ} ; \theta_{3}=18.92^{\circ} ; \ldots$.

From the relation Eq.(20) it also follows that the radiation intensity for a constant outgoing angle will vary with $a / d$. In Ref.6 this problem was analysed for the nonrelativistic case.

Using Eq.(2) and (11) we may now arrive at an azimuthal dependence of the $\mathrm{SP}$ radiation yield for the angles $1>>\xi^{2}>>\gamma^{-2}$ :

$$
\frac{d W_{s p}^{\perp}}{d \Omega} \approx \frac{\alpha}{\pi^{2}} \frac{2 \omega_{-1}}{\sin ^{2} \theta} \exp \left(-4 \frac{h}{a} \varphi_{\perp} \sqrt{\frac{\gamma^{-2}}{\sin ^{2} \theta}+\xi^{2}}\right) \times\left(\varphi_{\perp}^{2} \xi^{2}+\sin ^{2} \varphi_{\perp}\right) .
$$

It is obvious that in the case where $\sin ^{2} \varphi_{\perp}<<\varphi_{\perp}^{2}$ (i.e. near $\varphi_{\perp}=m \pi$ ) the azimuthal dependence (21) would have the minimum for $\xi=0$ and peak at $\xi \sim \frac{a}{2 h \varphi_{\perp}}>>\gamma^{-1}$. On the contrary, for parallel case distribution (17) has only one maximum at $\xi=0$. Fig.3 presents the calculations for both geometries. In the relativistic case one can always choose the geometry and the grating parameters such that the radiation would be concentrated near the plane normal to the grating. As a result, first, the radiation brightness $d W / d \Omega$ increases and, second, configuration of the would-be resonators and mirrors is simplified to an advantage as compared to that proposed in Ref.13, which may turn out quite useful assuming the construction of an SPE - based free electron laser.

The following should be noted in conclusion. All the experiments on SPE studies of which the author is aware have been performed with volume gratings, i.e. when a beam of virtual photons is reflected from a continuous surface deformed via a certain periodic law. For instance, in the case of a lamellar grating each unit cell of the latter contains two perpendicular and two parallel planes. Due to destructive interference the reflectivity $F$ of the grating element ( $\left|R_{-1}\right|^{2}$ in terms of Ref.4) is sufficiently below unity $\left(10^{-2} \div 10^{-3}\right.$, while for a grating of parallel strips separated by vacuum gaps, this quantity may reach a value of 4 (see Eq.(5)) and, therefore, the SPR intensity would grow in the same fashion (increasing by 2-3 orders). 
In the relativistic case the thickness is chosen such that $\gamma \lambda>>b$ at least in the infrared and millimeter range, where polished aluminum could be considered as an ideal conductor.

Thus, for our case the model proposed is going to be sufficiently valid. The deduction made by the authors of Ref.4 on the absence of advantages for the relativistic beams to be used as the SPR generators seems to us premature.

The author greatly appreciates the contribution by Prof.M.Ikezawa and Dr.Y.Shibata rendered through numerous fruitful discussions and stimulating criticism as well as the possibility of getting acquainted with their experimental results before publication. 


\section{References}

1. G.Doucas, J.H.Mulvey, M.Omori et al. Phys.Rev.Lett., $\underline{69}$, 1761(1992)

2. K.J.Woods, J.E.Walsh, R.E.Stoner et al. Phys.Rev.E., $\underline{74}$, 3808(1995)

3. K.Ishi, Y.Shibata, T.Takahashi et al. Phys.Rev.E, $\underline{51}$, R5212(1995)

4. O.Haeberle, P.Rullhusen, J.M.Salome et al.Phys.Rev.E, $\underline{49}$, $3340(1994)$

5. J.Walsh, K.Woods, S.Yeager. NIM A341, 277(1994)

6. B.M.Bolotovski, G.V.Voskresenskii. Sov. Phys. Uspekhi,11, 143(1968)

7. M.L.Ter-Mikaelian. High-Energy Electromagnetic Processes in Condensed Media (Wiley Interscience, New York, 1972)

8. M.J.Moran. Phys.Rev.Lett. $\underline{69}$, 2523(1992)

9. A.P.Kazantsev, G.I.Surdutovich. Sov.Phys.-Doklady, 7 , 990(1963)

10. X.Artru, G.B.Yodh, G.Mennessier . Phys.Rev.D,12, $1289(1975)$

11. G.M.Garibyan, L.A.Gevorgyan, C.Yang. Sov.Phys.JETP, $\underline{39}$, 265(1974)

12. Yu.N.Adischev, V.A.Verzilov, A.P.Potylitsin et al. NIM B44, 130(1989)

13. A.Gover, P.Dvorkis, U.Elisha. J.Opt.Soc.Am. B1, 723(1984) 


\section{Figure Captions}

Fig.1 Grating geometry with finite width strips :

a) - parallel and

b) - perpendicular configuration

Fig.2 Angular distribution of SPR intensity :

upper curve - geometry a),

lower curve - geometry b).

Fig.3 Azimuthal dependence of SPR intensity for polar angle

$\theta=70^{\circ}$ :

upper curve - geometry a),

lower curve - geometry b). 
a)

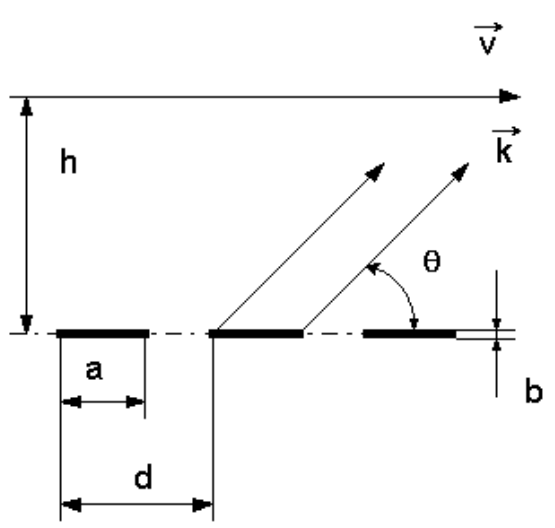

b)

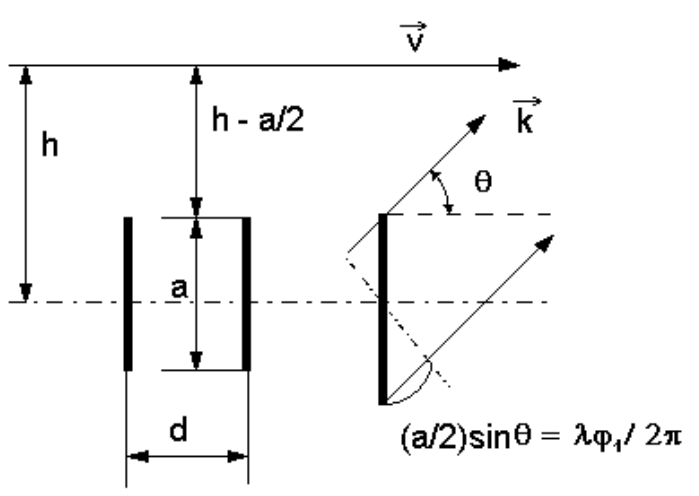

Fig. 1 


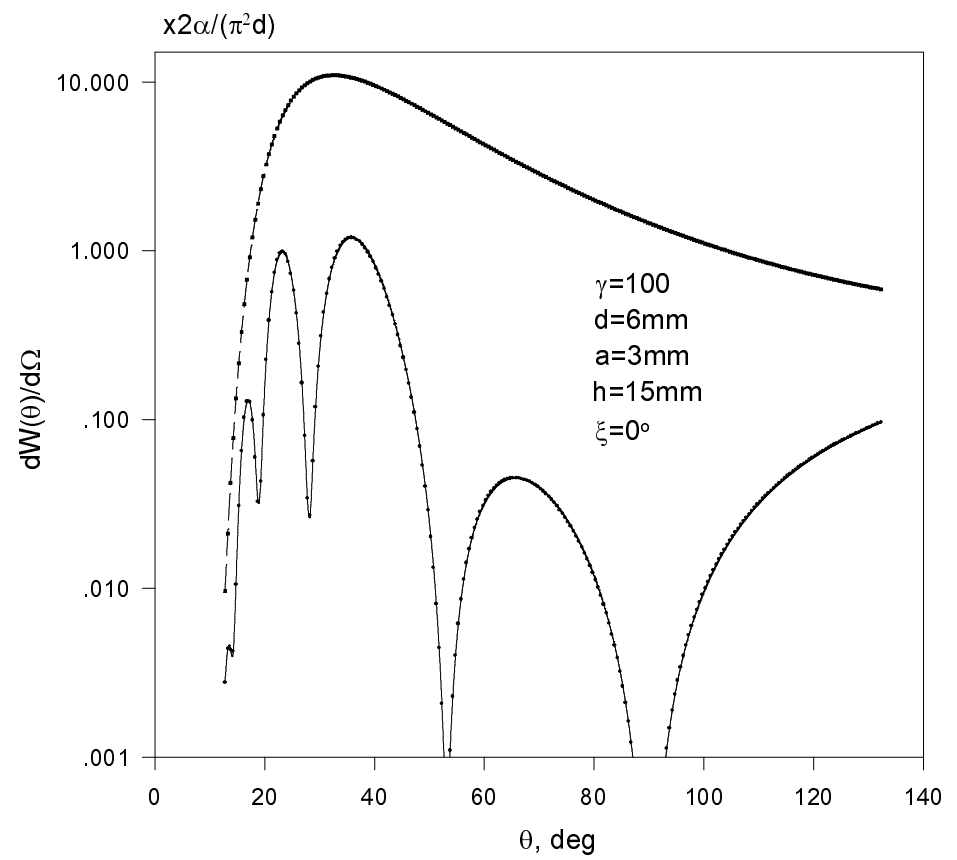

Fig. 2 


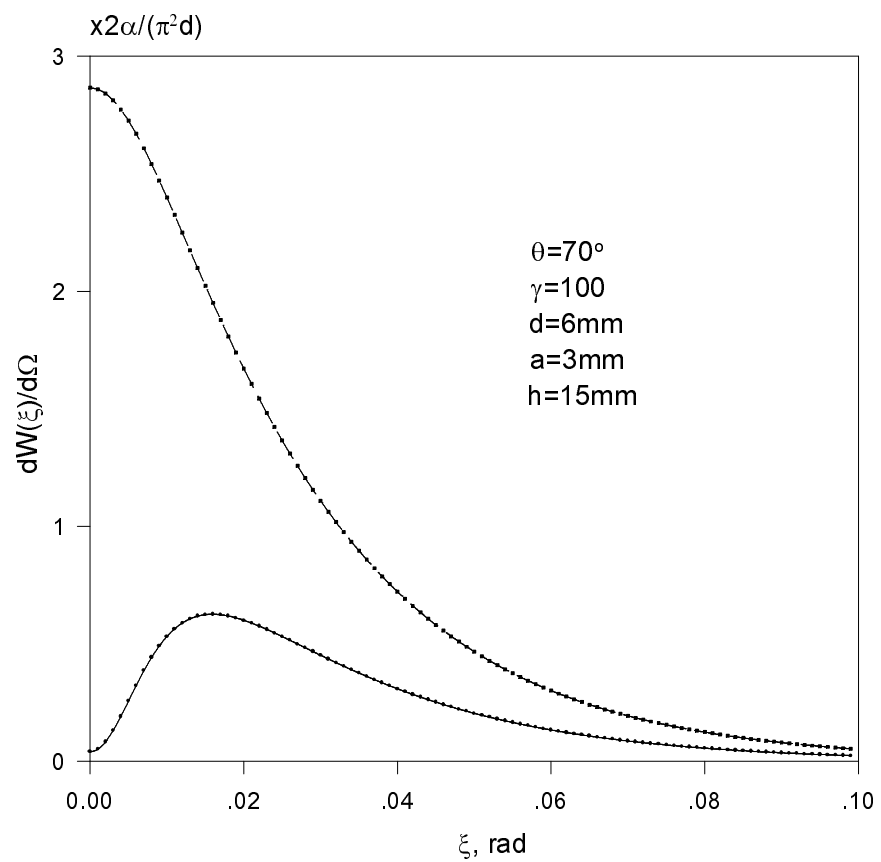

Fig. 3 